\title{
Propiedades térmicas y fisicoquímicas de pellets con fines energéticos elaborados a partir de residuos de aserradero
}

\section{Thermal and physiochemical properties of pellets with power aims made of sawmill residual product}

\author{
Pedro Casanova Treto \\ Instituto de Investigaciones en Ingeniería, Universidad de Costa Rica (UCR) \\ pcasanova2000@gmail.com \\ Kattia Solís \\ Escuela de Ingeniería en Biosistemas. Universidad de Costa Rica \\ kattia.solis@ucr.ac.cr, \\ Tonny Carrillo \\ tcarpani13@gmail.com
}

Recibido: 9 de febrero 2016

Aceptado: 8 de agosto 2016

\section{Resumen:}

Aserrín residual producto del aprovechamiento de la madera de las especies Pilón (Hyeronima alchorneoides) y Eucalipto (Eucalytus spp), fue empleado para elaborar pellets bajo diferentes condiciones de densificación. Se utilizó equipo experimental para determinar la conductividad térmica, difusividad térmica y el calor específico. Propiedades fisicoquímicas de los pellets obtenidos bajo las diferentes condiciones de densificación, tales como el contenido de cenizas y el poder calorífico fueron determinadas. El contenido de nitrógeno, cloro y azufre, presente en el material empleado para elaborar los pellets fue estimado. Se determinaron valores para la conductividad térmica entre los $0,253 \mathrm{~W} / \mathrm{m} \cdot \mathrm{K}$ y $0,279 \mathrm{~W} / \mathrm{m} \cdot \mathrm{K} ; 1,748 \mathrm{~m}^{2} / \mathrm{s}$ y $2,314 \mathrm{~m} / \mathrm{s}$ para la difusividad térmica, y en el caso del calor específico se determinaron valores entre los 3,019 $\mathrm{kJ} / \mathrm{kg} \cdot \mathrm{K}$ y $2,183 \mathrm{~kJ} / \mathrm{kg} \cdot \mathrm{K}$. El poder calorífico superior tuvo valores entre los $18907 \mathrm{~kJ} / \mathrm{kg}$ y 18 $960 \mathrm{~kJ} / \mathrm{kg}$, se determinó un contenido de cenizas del 1,31 \% en base seca. Finalmente, se determinó que el contenido de nitrógeno, cloro y azufre presente en la biomasa residual empleada, corresponde a un $0,1129 \%, 0,0592 \%$ y $0,0317 \%$ respectivamente. Se determinó una relación directa entre el aumento de la densidad aparente de los pellets y las propiedades térmicas. El poder calorífico y el contenido de cenizas no se vieron afectados de forma significativa por los tratamientos aplicados. El contenido estimado de N, Cl y S corresponde al esperado en la biomasa seleccionada. Se corrobora que los pellets estudiados poseen características dentro de los parámetros recomendados por las normas estudiadas.

\section{Palabras clave:}

Pellets-producción, energía biomásica-producción, pellets, aprovechamientos de residuos, biomasa forestal, aprovechamiento de la madera. 


\section{Summary:}

Sawmill residual product of Pylon (Hyeronima alchorneoides) and Eucalyptus (Eucalyptus spp) species was used to produce pellets under different conditions of densification. Experimental equipment was used to determine the thermal conductivity, thermal diffusivity and specific heat. Physicochemical properties of the pellets obtained under different conditions of densification, such as ash content and calorific value were determined. The content of nitrogen, chlorine and sulfur present in the material used to produce the pellets was estimated. Thermal conductivity values were determined between $0,253 \mathrm{~W} / \mathrm{mK}$ and $0,279 \mathrm{~W} / \mathrm{mK} ; 1,748 \mathrm{~m}^{2} / \mathrm{s}$ and $2,314 \mathrm{~m}^{2} / \mathrm{s}$ for the thermal diffusivity, and in the case of specific heat values between $3,019 \mathrm{~kJ} / \mathrm{kgK}$ and 2,183 $\mathrm{kJ} / \mathrm{kgK}$ were determined. The high heat values was between $18907 \mathrm{~kJ} / \mathrm{kg}$ and $18960 \mathrm{~kJ} / \mathrm{kg}$, an ash content of $1,31 \%$ on a dry basis was determined. Finally, it was determined that the content of nitrogen, chlorine and sulfur present in the residual biomass used, corresponds to $0,1129 \%$, $0,0592 \%$ and $0,0317 \%$, respectively. A direct relationship between increasing the bulk density of the pellets and the thermal properties was determined. The calorific value and ash content were not significantly affected by the treatments applied. The estimated content of $\mathrm{N}, \mathrm{Cl}$ and S corresponds to that expected in the selected biomass. Comparison of the properties of the pellets produced under the conditions studied -densification, against regulations-, showed acceptable results, entering these in terms of different categories of quality.

\section{Keywords:}

Pellets- production, biomass energy- production, pellets, waste management, forest biomass, uses of wood. 


\section{INTRODUCCIÓN}

Los pellets son el resultado de la densificación de mezclas de aserrín y otros subproductos de las operaciones de aprovechamiento de la madera. Con su fabricación se consigue una mejora en las características de almacenamiento y transporte, se reduce la emisión de polvos en su manejo, así como contribuye a obtener una mayor de cantidad de energía calorífica por unidad volumétrica y un eficiente control de la combustión. Los pellets se utilizan como sustituto directo del carbón y gas natural en la producción de calor y electricidad tanto para grandes industrias, como en pequeñas instalaciones $(1,2)$.

Debido a las grandes cantidades de pellets empleadas para aplicaciones energéticas a gran escala, así como por las características de este tipo de material biomásico densificado, se puede presentar auto-calentamiento espontáneo de estos durante el almacenamiento, lo que a su vez, dependiendo de las condiciones de temperatura, contenido de humedad, ventilación, y periodo de almacenamiento, eventualmente puede conducir a graves incendios espontáneos. Es por ello que es necesario conocer las propiedades térmicas de los pellets tales como la conductividad térmica, difusividad térmica y el calor específico, con el objetivo de hacer un diseño de infraestructura eficiente para su almacenamiento, y así proceder con la selección adecuada de los sistemas de enfriamiento y ventilación (3). Es necesario, entonces, determinar las propiedades térmicas de los pellets, en lugar de aplicar directamente las correlaciones reportadas para madera (4).

Respecto a la especificidad de cada propiedad, por definición la conductividad térmica está relacionada con la cantidad de calor que fluye a través de un material en función de un gradiente de temperatura que en este exista. Por su parte, la difusividad térmica indica qué tan rápido fluye dicha cantidad de calor a través de dicho material. Se sigue que, físicamente, estas dos propiedades térmicas se relacionan con la habilidad de un material para conducir calor, así como su destreza para almacenarlo; esto, a su vez, se relaciona con el calor especifico, que corresponde a la cantidad de calor necesaria para elevar la temperatura del material en $1^{\circ} \mathrm{C}$, por unidad de masa y sin cambio de estado (5).

Por otra parte, para una mejor aplicación de la biomasa como fuente energética, es clave no solo garantizar la oferta utilizando los residuos biomásicos disponibles y optimizar los procesos tecnológicos para el pretratamiento de la biomasa y su densificación, sino que también es fundamental cumplir con la calidad de estos biocombustibles de acuerdo a lo especificado en las normativas definidas para pellets con fines energéticos (6). Debido a esto es que se define una de las características energéticas más importantes para los biocombustibles: el poder calorífico, que denota la cantidad de energía calorífica producida por unidad de masa durante la combustión, por lo que a su vez esta propiedad distingue la calidad energética de los biocombustibles en razón de su capacidad calorífica específica (7). Otra de las propiedades de interés corresponde al contenido de cenizas del material combustible: estas son el subproducto remanente del proceso de combustión, por lo que conocer esta propiedad es esencial para escoger la tecnología de combustión y remoción de cenizas más eficiente, debido al riesgo de generación de capas de cenizas en las bases de los hornos, que, a su vez, conducen a un flujo de aire irregular generándose una combustión incompleta (menos eficiente), y a su vez aumenta las emisiones provocadas (2). El contenido de elementos como el 
nitrógeno, cloro y azufre, presente en material empleado para fabricar los pellets, son de los rubros normalmente regulados. Esto obedece a que durante las reacciones de combustión, los pellets tienden a generar compuestos cuyos efectos son la corrosión y deterioro de los equipos de combustión, tienen un alto grado de peligrosidad para la salud humana y, por último, y no menos importante, generan emisiones relacionadas con gases de efecto invernadero $(8,9)$.

Debido a esta problemática se plantea determinar las propiedades térmicas (conductividad térmica, difusividad térmica y calor específico) y fisicoquímicas (contenido de cenizas, nitrógeno, cloro, azufre y poder calorífico) de pellets con fines energéticos elaborados a partir de residuos de aserradero; además, evaluar el efecto de las condiciones de densificación experimentales sobre las propiedades determinadas; $y$, finalmente, comparar los resultados de las propiedades fisicoquímicas determinadas, respecto a lo estipulado en las principales normativas europeas para la calidad de pellets.

\section{MATERIALES Y MÉTODOS}

\subsection{Materiales y equipo de densificación}

Residuos de aserradero producidos en el aprovechamiento de la madera de las especies Pilón (Hyeronima alchorneoides) y Eucalipto (Eucalytus spp) fueron empleados para elaborar los pellets. El material residual colectado constó de partículas de aserrín y "burucha" en combinación de ambas especies; el contenido de humedad del material colectado fue del $35 \%$ b.h. El residuo fue triturado empleando un molino de martillos y cernido con un tamiz \#10 (apertura de $2 \mathrm{~mm}$ ). Finalmente el material fue secado a una temperatura de $60{ }^{\circ} \mathrm{C}$ hasta alcanzar un contenido de humedad del $8 \%$ b.h.

Para fabricar los pellets se empleó equipo experimental que funciona mediante un sistema cilindro-pistón, tal y como muestra la Fig. 1. Este equipo consta de un cilindro de $15 \mathrm{~cm}$ de largo y una perforación circular de $10 \mathrm{~mm}$ de diámetro en su eje longitudinal, el cual será el mismo diámetro resultante de los pellets. Un pistón del mismo diámetro de la perforación (10 mm) y $25 \mathrm{~cm}$ de largo, se introduce en la cavidad cilíndrica para comprimir el residuo. Este equipo se instaló en una prensa hidráulica cuyo vástago móvil imprime la fuerza al pistón y este al residuo. En la base de la unidad de peletizado se colocó una celda de carga conectada a su consola digital que mide e indica la fuerza aplicada al residuo; esta puede dar lecturas hasta los $50000 \pm 7 \mathrm{kgf}$. Al cilindro se le instaló un calentador eléctrico tipo abrazadera y una termocupla. La termocupla se introduce directamente en el cilindro metálico, y permite registrar la temperatura del cilindro mediante una consola digital. Esta última regula la temperatura que la abrazadera aplica al cilindro; la consola de la abrazadera/termocupla tiene una precisión de $\pm 1{ }^{\circ} \mathrm{C}$.

Se fabricaron pellets bajo dos tratamientos de densificación. Se elaboraron pellets de $10 \mathrm{~mm}$ de diámetro, aplicando al residuo una fuerza de compresión de $300 \mathrm{kgf}$ y una temperatura al cilindro de compresión de $90{ }^{\circ} \mathrm{C}\left(10 \mathrm{~mm}-300 \mathrm{kgf}-90{ }^{\circ} \mathrm{C}\right)$ obteniéndose pellets con una densidad aparente de $480,0 \mathrm{~kg} / \mathrm{m}^{3}$. Para el segundo tratamiento, los pellets se fabricaron a $10 \mathrm{~mm}$ de diámetro, el material se comprimió usando una 
fuerza de $500 \mathrm{kgf}$ y se aplicó una temperatura de $105^{\circ} \mathrm{C}\left(10 \mathrm{~mm}-500 \mathrm{kgf}-105^{\circ} \mathrm{C}\right)$. Se obtuvo una densidad aparente de $552,3 \mathrm{~kg} / \mathrm{m}^{3}$ para esta muestra de pellets. La totalidad de pellets se fabricaron empleando $2 \pm 0,1 \mathrm{~g}$ de aserrín. El tiempo de compresión para formar cada pellet fue de 60 segundos. Los pellets fueron fabricados en el taller de carpintería de la Unidad de Recursos Forestales, Instituto de Investigaciones en Ingeniería de la Universidad de Costa Rica.

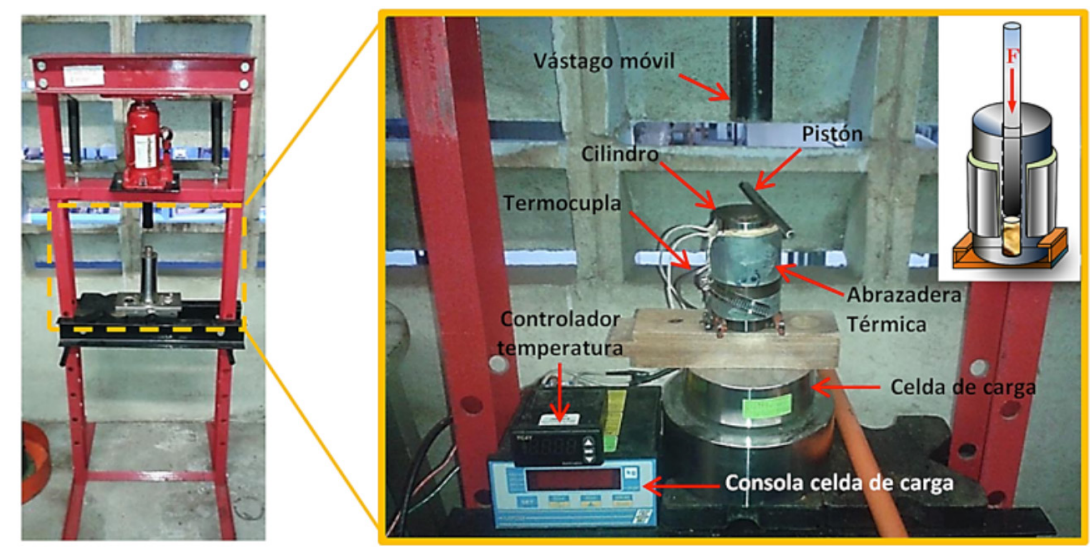

Figura 1. Equipo experimental empleado para elaborar los pellets a) Prensa hidráulica b) Cilindro - pistón (más accesorios)

\subsection{Determinación de las propiedades térmicas}

Se determinó la conductividad térmica, la difusividad térmica y el calor específico de las muestras de pellets empleando equipo experimental. Este equipamiento se adaptó según lo descrito por $(4,10)$. En la Fig. 2 se muestra un esquema general de los componentes que lo conforman.

El equipo funciona bajo el fundamento termodinámico de un cilindro semi-infinito. Está construido con tubo PVC de $10 \mathrm{~cm}$ de diámetro y $30 \mathrm{~cm}$ de largo; en sus dos extremos está recubierto con aislante térmico de "styrofoam" y sellado con tapones, uno de los cuales es removible con el fin de rellenar con muestra el cilindro. A lo largo del eje central del cilindro pasa una resistencia eléctrica de $30 \mathrm{~cm}$ de largo, que funciona como fuente de calor lineal; se asume la hipótesis que se da conducción de calor unidireccional y radial a partir de la fuente lineal de calor (11). Se aplicó a la resistencia un voltaje de 1,75 V y un consumo de corriente eléctrica de 1,50 A. El equipo se puso en funcionamiento dentro de una cámara tipo incubadora con temperatura constante de $23{ }^{\circ} \mathrm{C}$.

Las corridas experimentales del equipo consisten en llenar el cilindro de la muestra de pellets y activar la fuente de poder. Seguidamente se registra el cambio de la temperatura de la resistencia y diferentes puntos en el interior del cilindro durante 1,3 horas. La toma de temperaturas es realizada con 4 termopares; el termopar T0 registra el cambio de la temperatura de la resistencia, y otros tres termopares T1, T2, y T3 
registran la temperatura en puntos ubicados a una distancia radial de $1 \mathrm{~cm}, 2,5 \mathrm{~cm}$ y $4 \mathrm{~cm}$ respectivamente; un último termopar T4 registra la temperatura de la cámara. Finalmente, el cambio en los perfiles de temperatura con el tiempo para cada uno de los puntos estudiados (T0, T1, T2, y T3), se relaciona con la conductividad térmica, la difusividad térmica y el calor específico de la muestra, de acuerdo con las ecuaciones 1 , 2 y 3 respectivamente. Este procedimiento se realizó por triplicado para las muestras de pellets obtenidas en los dos tratamientos estudiados, con lo que se reportó el promedio. Este procedimiento se realizó en el Laboratorio de la Unidad de Recursos Forestales, Instituto de Investigaciones en Ingeniería de la Universidad de Costa Rica.

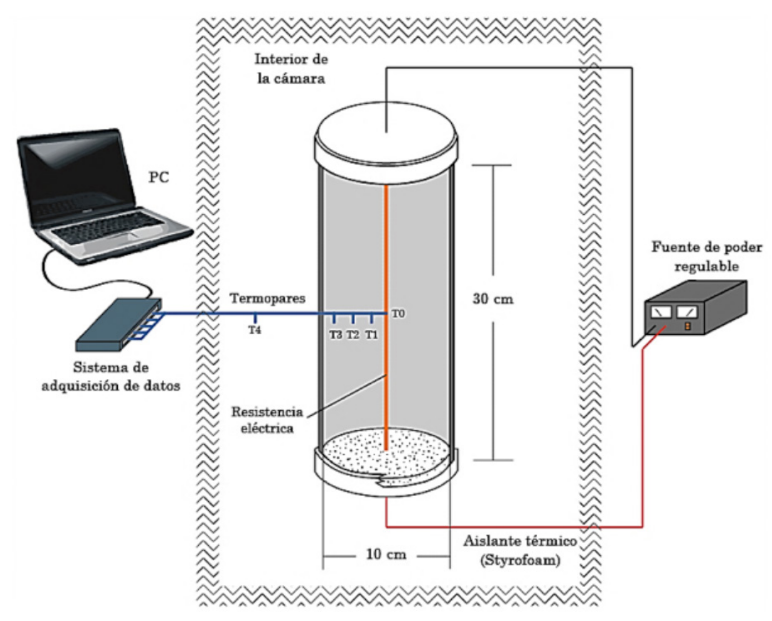

Figura 2. Esquema del equipo empleado para determinar las propiedades térmicas.

Para determinar la conductividad térmica se empleó la siguiente ecuación (5).

$$
k=\frac{q}{4 \pi L\left(T_{2}-T_{1}\right)} \ln \left(\frac{t_{2}}{t_{1}}\right)
$$

donde:

$k \quad$ es la conductividad térmica de la muestra $\left(\mathrm{W} \cdot \mathrm{m}^{-1} \cdot \mathrm{K}^{-1}\right)$

$q \quad$ es la potencia disipada por la resistencia (W)

$L \quad$ es la longitud del cilindro (m)

$T_{1}$ y $T_{2}$ son las temperaturas inicial y final de la resistencia respectivamente $\left({ }^{\circ} \mathrm{C}\right)$

$t_{1}$ y $t_{2}$ son los tiempos inicial y final respectivamente (s)

La difusividad térmica se determinó según (12): 


$$
\alpha=\left[\frac{e^{\left(\frac{4 \pi k \cdot \Delta T}{q}+y\right)}}{4 t}\right] r^{2}
$$

donde:

$\alpha \quad$ es la difusividad térmica de la muestra $\left(\mathrm{m}^{2} \mathrm{~s}^{-1}\right)$

$r \quad$ es la distancia radial de toma de lecturas (m)

$\Delta T \quad$ es el gradiente de temperatura a la distancia $r\left({ }^{\circ} \mathrm{C}\right)$

$k \quad$ es la conductividad térmica de la muestra $\left(\mathrm{W} \mathrm{m}^{-1} \cdot \mathrm{K}^{-1}\right)$

$q \quad$ es la potencia disipada por la resistencia (W)

$y \quad$ es la constante de Euler $(y=0,5772)$

$t \quad$ es el intervalo de tiempo de las mediciones (s)

Una vez determinada la conductividad y difusividad térmica de las muestras, se determinó de forma indirecta el calor específico de estas empleando la ecuación 3, según (5)

$$
c_{p}=\frac{k}{\alpha \rho_{a}}
$$

donde:

$C_{p} \quad$ es el calor específico de la muestra $\left(\mathrm{kJ} \cdot \mathrm{kg}^{-1} \cdot \mathrm{K}^{-1}\right)$

$k \quad$ es la conductividad térmica de la muestra $\left(\mathrm{W} \mathrm{m}^{-1} \cdot \mathrm{K}^{-1}\right)$

$\alpha \quad$ es la difusividad térmica de la muestra $\left(\mathrm{m}^{2} \cdot \mathrm{s}^{-1}\right)$

$\varrho_{a} \quad$ es la densidad aparente de la muestra $\left(\mathrm{kg} \cdot \mathrm{m}^{-3}\right)$

\subsection{Determinación de las propiedades fisicoquímicas}

Se determinó el contenido de cenizas de los pellets siguiendo el procedimiento establecido en la Norma CEN/TS 14775:2009. Este procedimiento se realizó en el Laboratorio de la Unidad de Recursos Forestales, Instituto de Investigaciones en Ingeniería de la Universidad de Costa Rica. Para el método empleado se registró la masa de una muestra de pellets superior a $1 \mathrm{~g}$ y se colocó en un horno tipo "mufla" a $250{ }^{\circ} \mathrm{C}$ durante 1,5 horas; seguidamente se aumentó la temperatura a $550^{\circ} \mathrm{C}$ y se mantuvo durante un periodo de 2,5 horas. Finalmente, se determina la masa de las cenizas obtenidas al final del procedimiento. Este procedimiento se realizó por triplicado para los pellets obtenidos en cada uno de los dos tratamientos de densificación aplicados.

El contenido de cenizas de la muestra expresado en porcentaje de la masa en base seca, se determinó empleando la ecuación 4 


$$
C_{b s}=\frac{100 \cdot m_{f}}{m_{i}} \cdot \frac{100}{\left(100-C H_{b s}\right)}
$$

donde:

$C_{b s} \quad$ es el contenido de cenizas de la muestra de pellets en base seca (\%)

$m_{i} \quad$ es la masa inicial de la muestra de pellets $(\mathrm{g})$

$m_{f} \quad$ es la masa final de las cenizas $(\mathrm{g})$

$\mathrm{CH}_{b s}$ es el contenido de humedad de la muestra en base seca (\%)

Se determinó el poder calorífico superior en tres muestras escogidas de forma aleatoria para cada unidad experimental, con lo que se reportó el promedio. Para determinar el poder calorífico superior (PCS) de las muestras de pellets, se empleó el método de la bomba calorimétrica, equipo marca "Parr Oxigen Bombs 1341". Este procedimiento se realizó en el Laboratorio de Fisicoquímica de la Escuela de Química de la Universidad de Costa Rica.

Para calcular el poder calorífico inferior (PCI) de las muestras, se restó al (PCS) el calor de condensación de la humedad presente en las muestras. Para esto se empleó la siguiente ecuación

$$
P C I=P C S-\left(C H \cdot L_{c}\right)
$$

donde:

PCI es el poder calorífico inferior de la muestra (kJ· kg -1)

PCS es el poder calorífico superior de la muestra (kJ· kg -1)

$\mathrm{CH}$ es el contenido de humedad de la muestra en base húmeda $(\mathrm{CH}=0,08)$ (todos los pellets fueron elaborados con aserrín al $8 \%$ de humedad, base húmeda)

Lc es el calor latente de condensación del vapor de agua liberado en la combustión de la muestra $(\mathrm{Lc}=2257 \mathrm{~kJ} \cdot \mathrm{kg}-1)$

La determinación del contenido de nitrógeno, cloro y azufre presente en la biomasa empleada para elaborar los pellets, se realizó en el Laboratorio de Química de la Atmósfera (LAQAT) de la Escuela de Química de la Universidad Nacional de Costa Rica.

Para el caso específico del contenido de azufre y cloro, se empleó un cromatógrafo de iones marca "Dionex 5000". Este equipo analiza muestras compuestas de la disolución obtenida del lavado de los residuos de la combustión de las muestras de pellets, procedimiento que se realizó empleando una bomba calorimétrica marca "IKA C200".

Para la determinación del contenido de nitrógeno en las muestras se empleó el método "Kjeldahl". Para este procedimiento, se realizó la digestión de la muestra de pellet con ácido sulfúrico y catalizadores (sulfato de cobre y sulfato de potasio); posteriormente, la muestra digerida se mezcló con hidróxido de sodio para seguidamente 
proceder a destilar el amonio presente en esta disolución, el cual fue capturado sobre ácido bórico con rojo de metilo como indicador. Finalmente, se realizó la titulación, determinándose el contenido de nitrógeno presente en la muestra analizada a partir de la cantidad de ácido sulfúrico empleada en la titulación.

\subsection{Incertidumbre de los resultados}

Para determinar la incertidumbre de los resultados de propiedades térmicas, del contenido de cenizas y del poder calorífico, se utilizó el programa Engineering Equation Solver (EES); más específicamente, se empleó la herramienta de propagación de incertidumbres (Uncertainty Propagation). Esta herramienta computacional estima la incertidumbre de los resultados de acuerdo al "error" o "incertidumbre estándar combinada", que asocia la precisión absoluta de los equipos utilizados y las ecuaciones empleadas para determinar cada propiedad. El software EES emplea la ecuación 6 para estimar la incertidumbre

$$
e_{R}=\left[\left(\frac{\partial F}{\partial X} \cdot \Delta_{x}\right)^{2}+\left(\frac{\partial F}{\partial Y} \cdot \Delta_{y}\right)^{2}+\left(\frac{\partial F}{\partial Z} \cdot \Delta_{z}\right)^{2}+\ldots\right]^{\frac{1}{2}}
$$

donde:

$$
\begin{array}{ll}
e_{R} & \text { es la incertidumbre (error) estándar combinada del resultado final. } \\
F & \text { es una función dependiente de las variables independientes } X, Y, Z \ldots \\
\Delta_{x}, \Delta_{y}, \Delta_{z} \ldots \text { son las incertidumbres absolutas de los equipos con que se midieron } \\
X, Y, Z \ldots
\end{array}
$$

La incertidumbre de los resultados en la determinación del contenido de $\mathrm{N}, \mathrm{Cl}$ y S, se reporta de acuerdo con el procedimiento para la estimación de esta, de acuerdo con el procedimiento en el Laboratorio de Química de la Atmósfera (LAQAT).

\subsection{Normativas de calidad de pellets consultadas}

La normativa de calidad de pellets, desarrollada principalmente en países europeos como Alemania (Normas DIN), Suecia (Normas SS) y Austria (Normas ÖNORM), así como la normativa de calidad de pellets desarrollada por el Comité de Normalización Europeo (CEN/TS 14961), en general tienen como objetivo garantizar la uniformidad de la biomasa densificada y reducir las barreras de mercado, creándose un flujo de producto en el que estos biocombustibles pueden ser objeto de comercio entre productores y usuarios sin importar países o regiones (13). En la Tabla 1 se muestran los principales parámetros definidos para evaluar la calidad de pellets, en las principales normas europeas. 


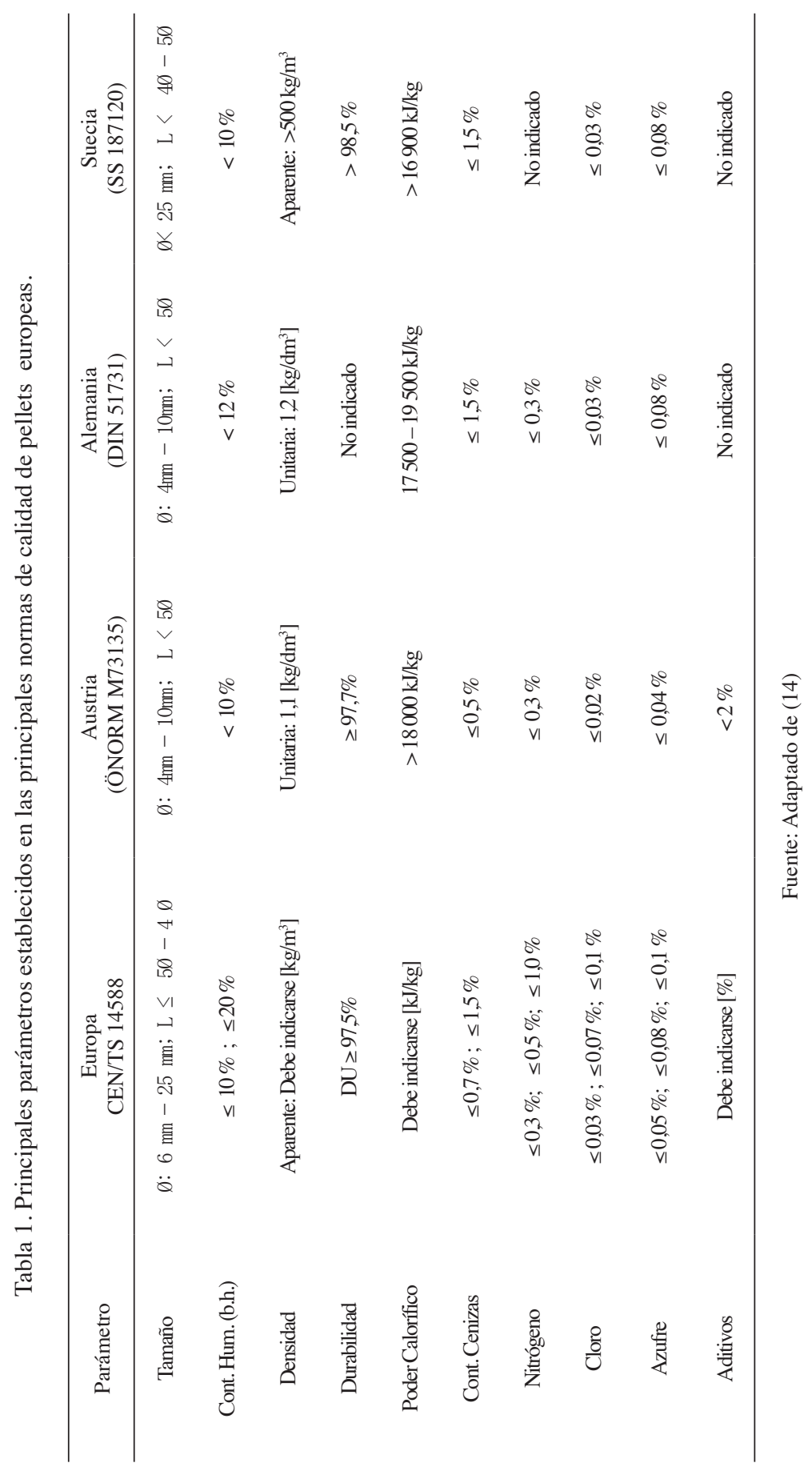




\section{RESULTADOS Y DISCUSIÓN}

\subsection{Propiedades térmicas}

La Figura 3 muestra un perfil de temperaturas obtenido de forma típica con el equipo empleado para determinar las propiedades térmicas. Los resultados de la Tabla 2 muestran la conductividad térmica calculada para las dos muestras estudiadas; se indican también la desviación estándar y la incertidumbre asociada a las mediciones para ambos casos.

Se muestra en la Tabla 2 que la conductividad térmica tuvo valores entre 0,255 $\mathrm{W} / \mathrm{m} / \mathrm{K}$ y $0,279 \mathrm{~W} / \mathrm{m} / \mathrm{K}$. para densidades de $480,0 \mathrm{~kg} / \mathrm{m}^{3}$ y $552,3 \mathrm{~kg} / \mathrm{m}^{3}$ respectivamente, que asimismo corresponden a los tratamientos de densificación (10 mm - 300 $\left.\mathrm{kgf}-90{ }^{\circ} \mathrm{C}\right)$ y $\left(10 \mathrm{~mm}-500 \mathrm{kgf}-105^{\circ} \mathrm{C}\right)$ respectivamente. Por lo que se observa que esta propiedad aumentó con la densidad aparente de las muestras analizadas.

Trabajos como el desarrollado por Guo et al., (4) reportan que la conductividad térmica de pellets hechos de aserrines estuvo entre $0,18 \mathrm{~W} / \mathrm{m} / \mathrm{K}$ y $0,25 \mathrm{~W} / \mathrm{m} / \mathrm{K}$. Por su parte, Sjöström \& Blomqvist (15) reportaron valores de conductividad unitaria para pellets (6 mm de diámetro y aproximadamente $12 \mathrm{~mm}$ de largo) de $0,266 \mathrm{~W} / \mathrm{m} / \mathrm{K}$. Fasina \& Sokhansanj (3), también reportaron valores similares.

De lo anterior se entiende que los valores determinados en este trabajo son superiores $(0,253-0,279 \mathrm{~W} / \mathrm{m} / \mathrm{K})$ a los reportados en la literatura consultada. Sin embargo, esta diferencia se sostiene sobre el hecho que la conductividad térmica varía también con la geometría específica de cada unidad de pellet, así como con el material empleado para elaborarlos (15); por lo que tomando el precedente de que las unidades aquí analizadas poseen dimensiones de $10 \mathrm{~mm}$ de diámetro, y alrededor de $20 \mathrm{~mm}$ de largo, los valores de $\mathrm{k}$ reportados en literatura, deben ser considerados como referencia del valor que se puede esperar en general. A pesar de esto, en los resultados aquí expuestos se observa que la conductividad térmica tiende a aumentar con la densidad aparente, de la misma forma Guo et al., (16) y Sjöström \& Blomqvist (15) observaron resultados similares. Además, se muestra un comportamiento similar en el caso de la difusividad térmica, en donde el valor determinado para $\alpha$ aumenta con la densidad de la muestra de pellets analizada, pasando de $1,748 \mathrm{~m}^{2} / \mathrm{s}$ con $\mathrm{Qa}=480,0 \mathrm{~kg} / \mathrm{m}^{3}$, a $2,314 \mathrm{~m}^{2} / \mathrm{s}$ con $\mathrm{Qa}=552,3$ $\mathrm{kg} / \mathrm{m} 3$. Lo anterior indica una mayor facilidad para transferir calor para muestras de pellets más densas, tanto en cantidad como en rapidez.

El calor específico calculado de forma indirecta empleando la ecuación 3, y a partir de los datos experimentales de conductividad y difusividad térmica, así como de densidad aparente, corresponden a $3,019 \mathrm{~kJ} / \mathrm{kg} \cdot \mathrm{K}$ y $2,183 \mathrm{~kJ} / \mathrm{kg} \cdot \mathrm{K}$ para las densidades de $480,0 \mathrm{~kg} / \mathrm{m}^{3}\left(10 \mathrm{~mm}-300 \mathrm{kgf}-90^{\circ} \mathrm{C}\right)$ y $552,3 \mathrm{~kg} / \mathrm{m}^{3}(10 \mathrm{~mm}-500$ $\mathrm{kgf}-105^{\circ} \mathrm{C}$ ) respectivamente. Se observa que el calor específico disminuye con la densidad aparente, lo cual es un resultado esperado, debido a que la conducta típica de sólidos y materiales a granulados, es que el calor específico disminuya con el aumento de la difusividad térmica de estos (15), lo que corresponde al comportamiento aquí determinado. 
Como se observa, la relación existente entre $\mathrm{k}$ y $\alpha$ es directa respecto al aumento en la densidad aparente de la muestra de pellets analizada. Caso inverso respecto al aumento de la densidad aparente se observó con el calor específico. Lo anterior complementando el hecho de que el comportamiento de las propiedades térmicas de una masa de pellets está interrelacionado también, con sus propiedades físicas, como es el caso de la densidad aparente y la geometría especifica del material analizado. De hecho, a la conductividad térmica determinada experimentalmente por métodos como el empleado en este trabajo, normalmente se le conoce como conductividad térmica efectiva, ya que se considera la masa de pellets a granel como una sola unidad con características isotrópicas de porosidad, densidad, contenido de humedad, etc. El caso de la difusividad térmica y del calor especifico guardan las mismas consideraciones.

Por otra parte, para materiales porosos como los pellets a granel, la conductividad es en realidad una combinación efectiva de la conductividad pura dentro de los pellets, la transferencia de calor por convección a la fase gaseosa e intercambio de radiación entre pellets (15). Y es por esto que para los pellets obtenidos de los dos tratamientos aplicados, se presentó un escenario de análisis desde un enfoque basado en la variación de las propiedades térmicas, debido a las diferencias observadas en la densidad aparente en las dos muestras de pellets estudiadas.

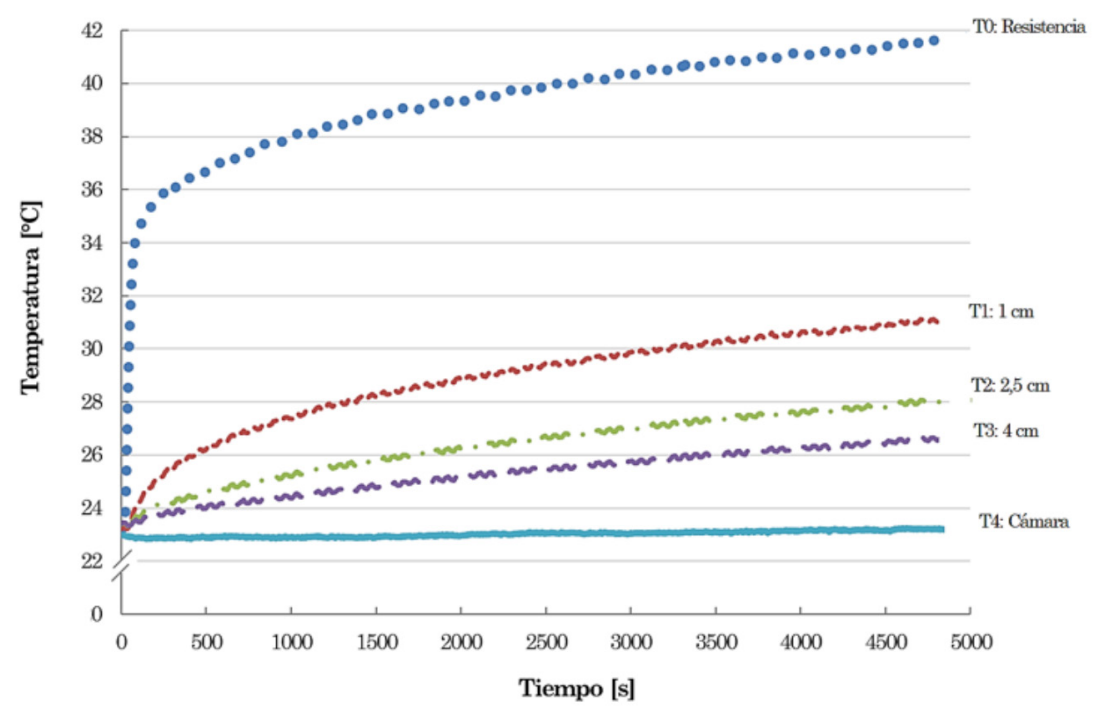

Figura 3. Perfil de temperatura obtenido con el equipo experimental construido para la determinación propiedades térmicas. Curva para una de las repeticiones del tratamiento $\left(10 \mathrm{~mm}-300 \mathrm{kgf}-90{ }^{\circ} \mathrm{C}\right) \mathrm{Qa}=480,0 \mathrm{~kg} / \mathrm{m}^{3}$.

\subsection{Propiedades fisicoquímicas}

\subsubsection{Contenido de cenizas}

Se presenta en la Tabla 3 los resultados obtenidos en la determinación del contenido de cenizas en porcentaje de la masa en base seca (b.s.) presente en los pellets, 


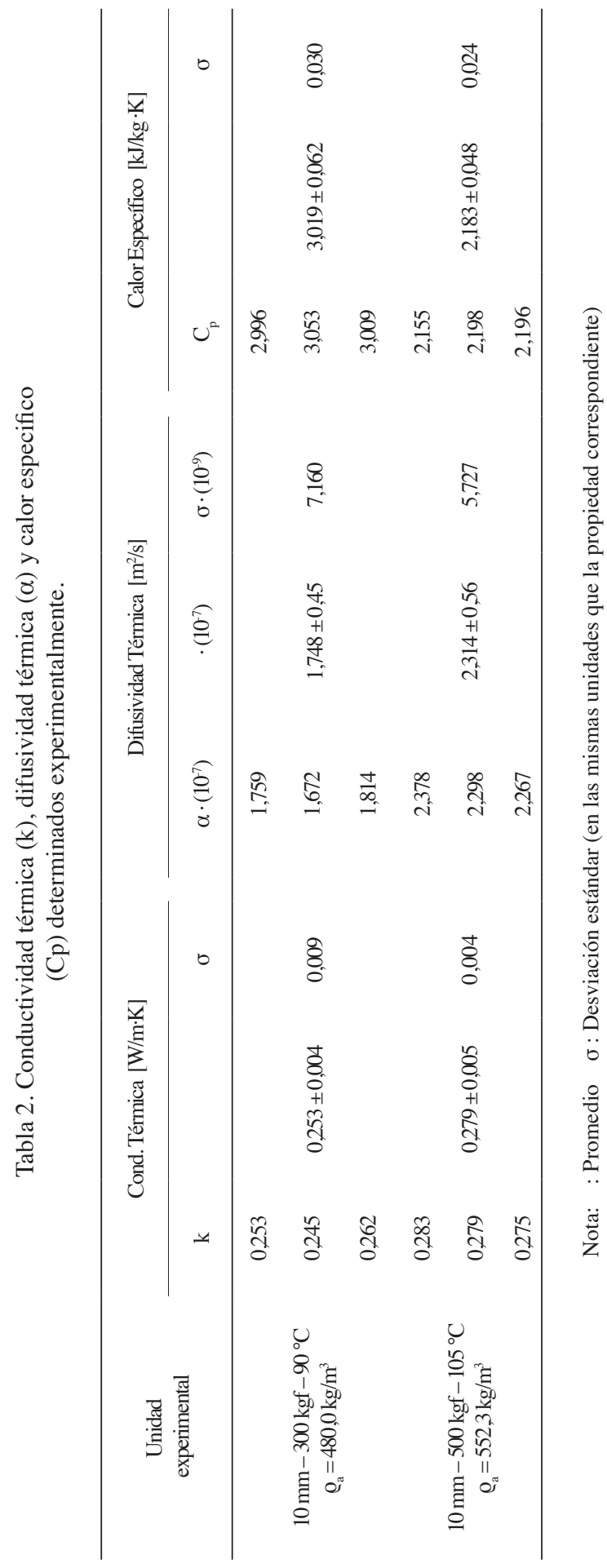


elaborados bajo los dos tratamientos de densificación aplicados, la incertidumbre ( \pm ) y la desviación estándar asociada a los resultados.

Los resultados obtenidos para el contenido de cenizas mostrados en la Tabla 3, muestra un promedio de $1,31 \%$ para el contenido de cenizas, y sin variación debido al efecto de los tratamientos de densificación aplicados a la biomasa residual al momento de fabricar los pellets. De hecho, el análisis de varianza entre medias de contenido de cenizas (Test SNK, con $\alpha=0,05)$, mostró que no existen diferencias significativas $(\mathrm{p}<0,05)$ entre los valores determinados para los pellets obtenidos de los dos tratamientos de densificación aplicados. Lo anterior, a su vez, advierte del efecto nulo de las condiciones de densificación sobre la variación del contenido de cenizas. Resultados similares fueron observados por Poddar et al. (17), quienes mostraron variación de propiedades fisicoquímicas seleccionadas (entre ellas el contenido de cenizas), con la aplicación de altas presiones de compresión y bajas temperaturas, para distintas biomasas.

Tabla 3. Contenido de cenizas en los pellets elaborados bajo los distintos tratamientos.

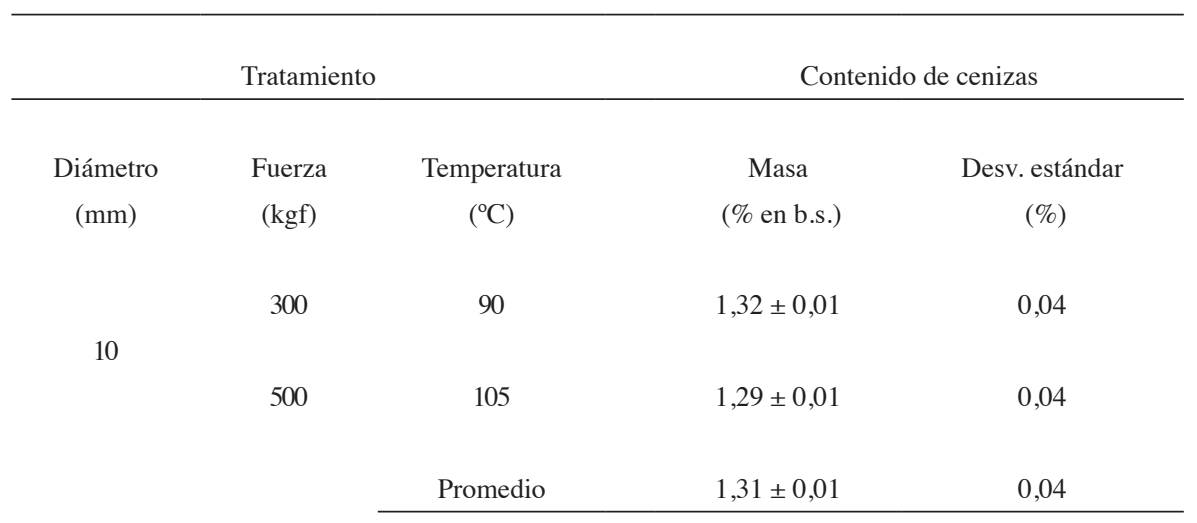

En respuesta a lo que las normativas europeas de calidad de pellets normalmente estipulan para el contenido de cenizas, se puede observar en la Tabla 1 que los valores establecidos para diferentes categorías de calidad corresponden a porcentajes debajo del $0,5 \%$ hasta $1,5 \%$, donde los valores inferiores normalmente corresponden a categorías de calidad superiores. El caso de los resultados obtenidos para la biomasa empleada en este trabajo apunta a que se obtiene un contenido de cenizas que corresponde a la categoría $1,5 \%$, determinándose una respuesta satisfactoria para este rubro en tres de cuatro normas presentadas en la Tabla 1, ya que el caso de la normativa de Austria (ÖNORM M73135) no permite contenidos de cenizas superiores al 0,3\% de la masa en base seca.

\subsubsection{Poder calorífico}

Los resultados obtenidos en la determinación del poder calorífico superior para los pellets elaborados bajo los distintos tratamientos estudiados, así como la incertidumbre asociada ( \pm ) y la desviación estándar respectiva, se muestran en la Tabla 4. 
El valor determinado experimentalmente con el método de la bomba calorimétrica corresponde al poder calorífico superior (PCS); sin embargo, en la Tabla 4 también se muestran resultados correspondientes al poder calorífico inferior (PCI), que responden al valor a cumplir en las normativas de calidad consultadas. Además, cabe recordar que el PCI obtenido es resultado de la resta al PCS del calor de condensación de la humedad presente en las muestras; esto de acuerdo con la ecuación 3. Es debido a esto que a los valores de PCS se les sustrajo $181 \mathrm{~kJ} / \mathrm{kg}$ correspondientes al $8 \%$ de humedad en base húmeda (b.h.) que tenía la biomasa residual empleada para fabricar los pellets; lo anterior cumpliendo con el rubro de contenido de humedad establecido comúnmente en las normativas consultadas ( $\leq 10 \%$ b.h.).

Tabla 4. Poder calorífico determinado en los distintos tratamientos aplicados.

\begin{tabular}{|c|c|c|c|c|c|}
\hline \multicolumn{3}{|c|}{ Tratamiento } & \multicolumn{3}{|c|}{ Poder Calorífico } \\
\hline $\begin{array}{l}\text { Diámetro } \\
(\mathrm{mm})\end{array}$ & $\begin{array}{c}\text { Fuerza } \\
\text { (kgf) }\end{array}$ & $\begin{array}{c}\text { Temperatura } \\
\left({ }^{\circ} \mathrm{C}\right)\end{array}$ & $\begin{array}{c}\text { Superior }(\mathrm{kJ} / \\
\mathrm{kg})\end{array}$ & $\begin{array}{l}\text { Inferior } \\
(\mathrm{kJ} / \mathrm{kg})\end{array}$ & $\begin{array}{c}\text { Desv. Estándar } \\
(\mathrm{kJ} / \mathrm{kg})\end{array}$ \\
\hline \multirow{2}{*}{10} & 300 & 90 & $18907 \pm 13$ & $18726 \pm 13$ & 40 \\
\hline & 500 & 105 & $18960 \pm 12$ & $18779 \pm 12$ & 39 \\
\hline & & Promedio & $18934 \pm 13$ & $18753 \pm 13$ & 40 \\
\hline
\end{tabular}

Por otra parte, se observa en la Tabla 4 que en los resultados del PCS se obtiene un promedio de $18934 \mathrm{~kJ} / \mathrm{kg}$, y en el caso del PCI, se tiene un promedio de 18753 $\mathrm{kJ} / \mathrm{kg}$. Los anteriores promedios para el PCS y PCI son definidos partiendo del resultado obtenido del análisis de varianza y de los resultados obtenidos en las repeticiones realizadas para determinar el poder calorífico para cada tratamiento (Test SNK, con $\alpha$ $=0,05)$, en donde se obtuvo como resultado que no existen diferencias significativas $(\mathrm{p}<0,05)$ entre las medias de PCS determinadas. Por lo que, de la misma forma que en el contenido de cenizas, se observa efecto nulo por parte de los tratamientos para influir sobre el PCS determinado.

Sin embargo, el efecto de una mayor fuerza de compresión está asociado a una mejora de las propiedades fisicoquímicas de densificados en forma de pellets (17). Lo anterior debido principalmente a una mayor activación de fuerzas intermoleculares, así como por una mayor remoción de humedad del material durante el proceso de compresión, lo que indirectamente influye, por ejemplo, sobre el poder calorífico determinado. A pesar de ello, esta tendencia no se refleja en los resultados encontrados en este trabajo, lo que en general apunta a un comportamiento no determinable para el poder calorífico, debido a la combinación de residuos de especies forestales empleada para fabricar los 
pellets, o, por otro lado, el rango empleado para los diámetros, fuerzas de compresión y temperatura, no permitió a dicho efecto manifestarse de forma significativa.

A pesar de la tendencia anteriormente descrita, los resultados obtenidos en el PCI son comparables con lo establecido en las normas de calidad consultadas en la Tabla 1, donde se observa que el PCI debe ser como mínimo igual o superior a los 16,9 MJ/ $\mathrm{kg}(16900 \mathrm{~kJ} / \mathrm{kg}) ; \mathrm{y}$, si se observan los resultados obtenidos para el PCI en la Tabla 4 y se toma el valor mínimo (18 $907 \mathrm{~kJ} / \mathrm{kg})$ o en su caso un promedio de todos los valores observados (18 $934 \mathrm{~kJ} / \mathrm{kg}$ ), se puede afirmar que la biomasa densificada empleada satisfizo lo estipulado para este rubro independientemente del tratamiento aplicado y la norma con la que se le compare.

\subsubsection{Contenido de nitrógeno, cloro y azufre}

Para el caso del contenido de N, Cl y S presente en la biomasa empleada, se muestran en la Tabla 5 los resultados para las repeticiones realizadas de los métodos de laboratorio utilizados. Se indica también la incertidumbre $( \pm)$ asociada a cada valor obtenido y la desviación estándar asociada.

En referencia a la Tabla 1.5, se observa que el contenido de nitrógeno, cloro y azufre son rubros a cumplir e indicar. Para el caso del nitrógeno se tiene que el contenido debe estar por debajo del 0,3\% para categorías de pellets superiores, y se llegan a aceptar porcentajes de nitrógeno de hasta un $1 \%$. Los resultados obtenidos muestran un contenido de nitrógeno promedio del 0,1129 \%, por lo que la biomasa seleccionada cumple con este rubro para cualquiera de las normas de calidad consultadas. Por su parte, el contenido de cloro promedio estimado corresponde a un $0,0592 \%$, valor que está muy cercano al valor estipulado para las categorías de calidad más exigentes $(\leq 0,03 \%)$; sin embargo, se puede ver que el valor estimado cumple con las categorías que aceptan contenidos de cloro menor o igual a $0,07 \%$.

A pesar de esto, en general se puede afirmar que la biomasa estudiada posee contenidos de este elemento en cantidades permisibles. En el caso del porcentaje de azufre presente en el material, se estimó un contenido promedio de $0,0317 \%$, valor que responde de forma satisfactoria al $0,04 \%$ o $0,05 \%$ normalmente estipulado como mínimo para cumplir con este rubro, en todas las normativas de calidad. Por tanto, se puede concluir que en general la biomasa seleccionada para densificación, responde de forma adecuada a los contenidos máximos para ser aceptada en las categorías de calidad más exigentes.

\section{CONCLUSIONES}

Tanto la conductividad térmica como la difusividad térmica guardan una relación directamente proporcional con el aumento de la densidad aparente de los pellets. Caso contrario del calor específico, que disminuyó para mayores densidades aparentes observadas para los pellets.

El contenido de cenizas fue determinado satisfactoriamente, concluyéndose que los tratamientos aplicados durante la densificación no tienen influencia alguna sobre esta propiedad. De la misma forma, no hubo variación significativa entre los valores de poder 
Tabla 5. Resultados de la determinación del contenido de N, Cl y S.

\begin{tabular}{|c|c|c|c|c|c|c|}
\hline \multirow{2}{*}{ Repetición } & \multicolumn{2}{|c|}{ Nitrógeno } & \multicolumn{2}{|c|}{ Cloro } & \multicolumn{2}{|c|}{ Azufre } \\
\hline & $\%$ Masa & $\pm(\%)$ & $\%$ Masa & $\pm(\%)$ & $\%$ Masa & $\pm(\%)$ \\
\hline 1 & 0,1163 & 0,0036 & 0,0571 & 0,0008 & 0,03850 & 0,00021 \\
\hline 2 & 0,1153 & 0,0040 & 0,0614 & 0,0007 & 0,04201 & 0,00025 \\
\hline 3 & 0,1124 & 0,0039 & 0,0629 & 0,0008 & 0,03204 & 0,00026 \\
\hline 4 & 0,1122 & 0,0039 & 0,0584 & 0,0008 & 0,02358 & 0,00025 \\
\hline 5 & 0,1109 & 0,0039 & 0,0638 & 0,0008 & 0,02226 & 0,00024 \\
\hline 6 & 0,1107 & 0,0038 & -.- & -- & --- & --- \\
\hline Desv.Est. (\%) & 0,0023 & --- & 0,0029 & -- & 0,0088 & --- \\
\hline Promedio & $\mathbf{0 , 1 1 2 9}$ & $\pm 0,0039$ & 0,0592 & $\pm 0,0008$ & 0,0317 & $\pm 0,00024$ \\
\hline
\end{tabular}

calorífico superior para cada tratamiento, por lo que se determinó que los tratamientos aplicados durante la densificación, no tienen efecto sobre esta propiedad fisicoquímica. Además, el poder calorífico inferior y contenido de cenizas determinado satisficieron lo estipulado en las normas de calidad consultadas.

Se estimó de forma satisfactoria el contenido de elementos químicos de interés presentes en el material empleado para fabricar los pellets, determinándose que estos contenidos cumplen con los valores máximos, acorde a lo estipulado para cada rubro, en las normativas de calidad de pellets consultadas.

En general, se concluye que la biomasa empleada, los rangos estudiados de diámetro, fuerza de compresión y temperatura de peletizado, dan como resultado pellets con propiedades fisicoquímicas que se ajustan de forma apropiada a los valores estipulados en las normativas de calidad pellets consultadas.

\section{REFERENCIAS}

1. Liu Z, Liu X, Fei B, Jiang Z, Cai Z, Yu Y. The properties of pellets from mixing bamboo and rice straw. Renew Energy. Elsevier Ltd; 2013;55:1-5.

2. Carroll JP, Finnan J. Physical and chemical properties of pellets from energy crops and cereal straws. Biosyst Eng [Internet]. 2012 Jun;112(2):151-9. Available from: http://linkinghub.elsevier.com/retrieve/pii/S1537511012000608 
3. Fasina O, Sokhansanj S. Bulk thermal properties of alfalfa pellets. Can Biosyst Eng. 1995;37(2):91-5.

4. Guo W, Lim J, Sokhansanj S, Bi X, Melin S. Determination of Thermal Conductivity of Wood Pellets Using Line Heat Source Method. In: 2009 Reno, Nevada, June 21 - June 24, 2009. St. Joseph, MI: American Society of Agricultural and Biological Engineers; 2009.

5. Ramaswamy H, Vijaya Raghavan G, Chakraverty A, Mujumdar A, editors. Handbook of Postharvest Technology [Internet]. CRC Press; 2003. Available from: http://www.crcnetbase.com/ doi/book/10.1201/9780203911310

6. Serrano C, Monedero E, Lapuerta M, Portero H. Effect of moisture content, particle size and pine addition on quality parameters of barley straw pellets. Fuel Process Technol [Internet]. 2011 Mar;92(3):699-706. Available from: http://linkinghub.elsevier.com/retrieve/pii/ S0378382010003942

7. Telmo C, Lousada J. Heating values of wood pellets from different species. Biomass and Bioenergy. Elsevier Ltd; 2011;35(7):2634-9.

8. Obernberger I, Brunner T, Barnthaler G. Chemical properties of solid biofuels - significance and impact. Biomass and Bioenergy. 2006;30(11):973-82.

9. Obernberger I, Thek G. The Pellet Handbook. London, UK: Earthscan; 2010.

10. Bitra VSP, Banu S, Ramakrishna P, Narender G, Womac AR. Moisture dependent thermal properties of peanut pods, kernels, and shells. Biosyst Eng [Internet]. 2010

Aug;106(4):503-12. Available from: http://linkinghub.elsevier.com/retrieve/pii/ S1537511010001236

11. Incropera FP, DeWitt DP, Bergman TL, Lavine AS. Fundamentals of Heat and Mass Transfer. Incropera FP, Incropera FPFOH, Transfer M, editors. Water. John Wiley \& Sons. Vol 6, Dekker Mechanical Engineering. 2007. 997 p.

12. Moura J, Devilla I, Martins D. Seminário de Iniciação Científica. In: Propriedades térmicas dos grãos de quinoa (Chenopodium quinua, Wild). Goiás, Brasil; 2010. p. 1-6.

13. Cabral J, Tatiane D, Braga M, Mitiko M, Behling C, Dias P, et al. Produção de briquetes e péletes a partir de resíduos agrícolas, agroindustrais e florestais. Brasil; 2012.

14. García-Maraver A, Popov V, Zamorano M. A review of European standards for pellet quality. Renew Energy [Internet]. Elsevier Ltd; 2011;36(12):3537-40. Available from: http:// linkinghub. elsevier.com/retrieve/pii/S0960148111002394

15. Sjöström J, Blomqvist P. Direct measurements of thermal properties of wood pellets: Eleva-ted temperatures, fine fractions and moisture content. Fuel. 2014;134:460-6.

16. Guo W, Lim CJ, Bi X, Sokhansanj S, Melin S. Determination of effective thermal conducti-vity and specific heat capacity of wood pellets. Fuel. Elsevier Ltd; 2013;103:347-55.

17. Poddar S, Kamruzzaman M, Sujan SM a., Hossain M, Jamal MS, Gafur M a., et al. Effect of compression pressure on lignocellulosic biomass pellet to improve fuel properties: Higher heating value. Fuel. Elsevier Ltd; 2014;131:43-8. 